\title{
Application of Artificial Neural Network to ANPR: An Overview
}

\section{Harish Paruchuri}

Big Data Engineer, Vintech Solutions, Inc., 9715 Olive Blvd, Olivette, MO 63132, USA

Corresponding Contact:

Email: harishparuchuri9999@gmail.com

\begin{abstract}
Vehicle owner documentation and traffic flow mechanism have contributed to a major issue in each country. From time to time it turns out to be challenging to detect car owners who fault traffic regulations. Hence, it of interest to us to investigate designs for automatic number plate detection structure as a clarification and proffer solution to this issue. There are several automatic number plate detection or recognition structure existing today. The structure is according to diverse methods nonetheless automatic number plate recognition is still a difficult job as many of the parameters such as a fast-moving vehicle, non-uniform car number plate, the language used in writing the vehicle number and various lighting situations may hinder 100\% detection rate. Many of the structure-function underneath these boundaries. This paper review diverse methods of automatic number plate recognition considering success rate, picture size, and processing time as factors. However, automatic number plate detection is recommended for traffic regulating agencies.
\end{abstract}

Keywords: Automatic Number Plate Recognition (ANPR), Character partitioning, Traffic flow, number plate

$12 / 31 / 2015$

Source of Support: Nil, Conflict of Interest: None Declared

This article is is licensed under a Creative Commons Attribution-NonCommercial 4.0 International License.

Attribution-NonCommercial (CC BY-NC) license lets others remix, tweak, and build upon work non-commercially, and

although the new works must also acknowledge \& be non-commercial.

\section{INTRODUCTION}

Over the years, one of the capable and essential techniques for vehicle monitoring and tracking has been automatic number plate recognition (ANPR) or License Plate Recognition (LPR). ANPR has been useful at numbering public places for providing satisfaction to some of the systems such as traffic safety enforcement, computerized device text assortment (You-Shyang and Ching-Hsue, 2010). Vehicle park structure (Anton and Ariff, 2008) and computerized car parking organization (Donepudi, 2014b). Automatic number plate recognition algorithms are commonly classified into 4 phases namely:

- Car picture capturing

- Numeral plate recognition

- Character categorization, and

- Character detection 
Currently, the number shield recognition and detection meting out time is not more than fifty ms (Christos et al., 2008) in several structures. The accomplishment of the $4^{\text {th }}$ phase is functions of the 2nd and 3rd phase are capable of locating the number shield of the vehicle and splitting every character.

These structures pursue diverse methods to spot the number shield of the vehicle and subsequently to extort the number shield of the vehicle from the picture. On the whole of the automatic number plate recognition structure are according to a usual method in the vein of artificial neural networking (ANN) (You-Shyang and Ching-Hsue, 2010; Chistos et al., 2008; Erdinc et al., 2011; Neogy \& Paruchuri, 2014; Roy and Ghoshal, 2011; Kaushik et al., 2012; Lucjan, 2012), Probabilistic neural network (PNN) (), optical character recognition (OCR) (Christos et al 2008; Jian et al., 2008; Xin and Guoliang, 2009; Anton and Ariff, 2008; Roy and Ghoshal, 2011; Lakshmi Narayana et al., 2012). Salient characteristic (Zhen-Xue, 2009), MATLAB (ChJaya et al., 2011), configurable technique (Lakshmi Narayana et al., 2012; Kaushik et al., 2012), sliding directed casement (Ying, 2011), support vector machine (Donepudi, 2014a), inductive learning (Wenjing et al., 2007), region oriented (Feng, 2008), color categorization (Morteza and Seyed, 2011), fuzzy oriented algorithm (Mei-sen et al., 3008; Movva et al., 2012; Kaushik et al., 2010), extent invariant characteristic transform (Francisco et al., 2012), trichromatic picturing, least-square technique (Cynthia et al., 2011), online authorize plate harmonizing oriented on biased revise remoteness (Ballard, 1981) and separate color features (Shen-Zheng and Hsi-Jian, 2007).

Authorize plate reader is described by (Prathamesh et al., 2009). Although, most of the researchers aimed at enhancing the quality of the image from the low-quality picture by applying a method known as tremendous verification (Suresh et al., 2007; Vadlamudi, 2015). According to Lucjan (2012) quality assessment of visual and automatic number plate recognition is properly documented.

The objective of the study is to survey automatic number plate recognition. The methods and each phase of ANPR.

Number shield recognition algorithms descend in many class-oriented on diverse methods. To recognize car number shield sequent parameters ought to be measured:

- Shield length: a shield can be of diverse dimension in a car picture.

- Shield setting: a shield can be situated wherever in the car.

- Shield conditions: A shield can include diverse conditions colors oriented to the car brand. For instance, a management car number shield may contain diverse conditions than erstwhile municipal cars.

- Fasten: A shield might contain fasten and that possibly will be measured as nature. A number shield can be isolated utilizing the picture partition technique. There are several picture partition techniques accessible in a variety of manuscripts. Considering the majority of the techniques picture binary is applied. A number of researchers utilize Otsu's technique for picture binary to exchange color picture to black size picture.

A number of shield partition algorithms are oriented on the color partition. An investigation of authorizing shield position according to color partition as asserted by Yang et al. (2011). 


\section{Attributes for Artificial Neural Network}

\section{Picture Linearization}

The picture binary or linearization is a phenomenon that changes a picture to a gray mode. In this technique, a definite verge is selected to categorize particular pixels as grey and particular pixels as white. But the major issue is how to select the right verge value for a certain picture. Adopted verge can be applied to surpass this issue. A verge can be chosen by operator guide or it can be chosen by an algorithm a computerize which is called as computerizing verge referred to as computerizing verge.

\section{Edge recognition}

Edge recognition is an essential technique for the isolation of the characteristics. Commonly, the task outcome of using edge recognition of algorithm is a device borderline with networking curves. Although this technique is very challenging to be applied especially to composite pictures as it may give rise to devise borderline with techniques, a range of manuscripts described the process for shield recognition. Even though the majority of the processes described in this paper use many methods, it is unrealistic to carry type-wise descriptions.

Diverse number shield partitioning algorithms are highlighted below:

According to Christos et al. (2008) for rapid recognition of the area of concentration a method known as descending concentric casement can be deployed. It comprises 2 phase concentric casements progressing from the higher left angle of the picture.

The arithmetical evaluations in both casements were computed according to the partition regulation that states, the proportion of the median or mean in the 2 casements that surpasses a verge, which is determined by the then central pixel of the casements is measured to be part of the rapid recognition of area of concentration. The 2 casements stop descending after the complete picture is inspected. The verge value may be determined according to a trial and blunder basis.

The relationship module analysis in addition to containing the in general accomplishment rate of ninety-six percentage. The research was accepted out on Pentium 4 system. The majority of the number shield recognition algorithms are under many but not linked angles. Divese edge recognition algorithm or uses such as Sobel, any-Deriche and Canny, disparity Prewitt and Roberts snappy are applied for edge recognition.

\section{Hough makeover}

It is a characteristic isolation method formerly applied for queue recognition. Its applications are presently extended to position search of random shapes such as oval or circle. The innovative algorithms were publicized by Ballard (1981).

\section{Blob exposure}

Blob exposure is applied mostly in detecting regions or points that be at variance in vividness or color as matched to the environment. The principal goal of deploying this method is to discover approving points or regions that are undetected by the edge recognition or angle recognition algorithm. Several blod exposures include Laplacian of Gaussian, diverse Gaussians, maximally stable external point or region, determinant of Hessian, and typical curving oriented point recognizer. 


\section{Linked module testing}

Linked module testing or blob isolation is a method to distinctively tag subsets of linked or related modules or elements according to an assigned heuristic. It glands binary picture and marks or tags pixel per connectivity situation of present pixel-like North, North-West, North-East, and West of the most recent pixel (connectivity), 4-connectivity is applied for simply north and west fellow citizen of the present pixel. The algorithm provides recovered act and it is extremely applicable for computerized picture test. This technique might be applied in shield partition and quality partition.

\section{Statistical morphology}

Statistical morphology is oriented on a set theory, topology, pattern theory, and arbitrary functions. It is generally relevant to the digital picture but also, might be applied in other spatial systems. At the outset, it was urbanized for dealing out binary pictures and after that complete for dispensation black and white scale quality and pictures. It comprises vital users like dilation, erosion, opening, and closing.

\section{METHODS}

To achieve the objective of this study, we attempt to review selected pieces of literature to address automatic number plate detection. This approach will us discuss common techniques deployed by the different agency in trail crime or theft. Besides the techniques discussed under the literature review, we will be reviewing related literature to address the best and acceptable method used.

\section{Results AND Discussion}

The techniques described in the foregoing segments are usual approaches for shield recognition. Other than the techniques mentioned here, several researchers have documented approaches for shield recognition. As the majority of the approaches mentioned in different papers deploy many techniques, it is impossible to do class-wise descriptions. Number shields section algorithms are described in this section.

According to Christos et al. (2008) for rapid recognition of area of significance, a method known as decreasing concentric casement is designed. It is a 2 phase technique that comprises 2 concentric casements touching from the higher left angle of the picture. Then mathematical dimensions in both casements were computerized according to the section regulation with the intention of if the proportion of the median or mean in the 2 casements exceeds a verge, which is laid down by then the innermost pixel of the casements is measured to be in the right position to the rapid recognition of position. The 2 casements bring to an end decreasing after the complete picture is scanned. The verge value may be determined according to a trial and error standpoint. The associated element test is also applied to contain complete success of ninety-six percentage. The testing was performed with Pentium IV at $3.0 \mathrm{GHz}$ with random access memory of 512-MB and take $111 \mathrm{~ms}$ of meting out moment for number shield partition.

An additional sliding concentric window-oriented structure is accessible according to Kaushik et al. (2012) for positioning the Korean shield. Subsequent to using a sliding concentric window on car picture researchers deployed HSI pigment design for color authentication and then incline was adjusted utilizing least-square appropriate with making corner offsets. The dimension amid the camera and car ranges from 7 to 3 meters. Shen-Zheng and Hsi-Jian (2007) discussed the cascade structure applied for designing a 
rapid algorithm for real-time car number shield recognition. This system provides a solid frame exposure of components deployed to the partition number shield. This component consists of 3 stages;

- Generation of shield area - applicants that is conversant of refusing non-shield area through gradient properties.

- Isolation of composite shield area that consists of 3 phases to detect shield area and decline no-shield areas.

- Shield authentification is applied to ensure that there is no non-shield area are isolated in the proceeding phase.

The research was performed on a 3-GHz Intel Pentium IV private PC. To identify multistyle number shields a configuration technique is projected by Ujwala et al. (2012). When it has to do with identifying diverse number shield style, an operator can

For detecting different styles of arranging the algorithm by varying variables figure in the number shield recognition algorithm. Ujwala et al. (2012) define the 4 variables as;

- Plate revolving angle - to revolve number at a particular corner plate when it is distorted.

- Character line digit - to detect whether characters are stretched in other than a line or pictures. It is a 7 stage process to isolate the number shield with no backdrop picture from the car image.

- Detection models- it has to do with identifying or detecting whether the number shield consists of alphabets or digits and alphabets or alphabet, symbols, and digits.

- Character styles - to categorize the number of shield characters according to their kind. For instance, symbols can be denoted as S, alphabets can be denoted as A and digits can be denoted as D.

Zhen-Xue et al. (2009) discussed the salient features associated with the technique deployed in isolating vehicle number shields. Some of the salient features include shapes, color, and texture. Zhen-Xue and co-author deployed Hough transform to recognize the horizontal and vertical line from rectangular-like car number shield and then processed the number by converting green, red, blue to tone intensity dispersion. Lastly, the number shield is partitioned. The algorithm deployed in the execution work with a PC with the following configuration; Pentium-4, processing speed of about 2.26-GHz, and random access memory of 1GB using MATLAB.

Ying (2011) reported enhancing the Bernsen algorithm method used for license shield location detection. This algorithm is best for situations such as irregular illumination and especially for shadow elimination. The author deployed global and local Otsu together with the degree of difference of local verge binary techniques for good precision. Working with this algorithm, the shadow is eradicated, and the license shield can be successfully recognized, which was not achievable with conventional Bernsen algorithms. The author performed the test on a Window XP operating system with a $1.8 \mathrm{GHz}$ Intel Core processing unit with random access memory of 1.5 GB. This algorithm is developed with Visual C++. Hui and Bing (2011) propose how to locate number plates in Chinese and the technique can help one in detecting both horizontal and vertical numbers. Hui and Bing transformed vehicle pictures into the black and white scale and then used automatic binary through MATLAB. These authors did not provide further information on how to detect number plate recognition, rather they claimed to record an average detection rate of 0.8 seconds. 


\section{Character Partitioning}

Once the number plate is located, characters are investigated for more process. When it comes to plate partitioning there are many techniques handy for determining character partitioning. Although many techniques are found in more than one class, it is impossible to discuss categories accurately. Many techniques like picture binary and connected component testing which we have discussed above can also be deployed to character partitioning.

Christos et al. (2008) proposed that applicants' area is shaped in $78 \times 228$ pixels utilizing bi-cubic outburst and also subjected to sliding concentric window for partitioning. They also deployed a verge value of 0.7 for improvement of outcome. Once the character partition procedure, each letter is resized to the pixel of $9 \times 12$. According to Prathamesh et al (2009) stated that peak to valley and blob coloring techniques are appropriate for a number plate in India. Their suggested picture scissoring algorithm in which a numbered shield is uprightly taken and scissor at the line where there is no white scale and this information is saved in the environment. When it has to do with more than one matrix, a bogus environment is rejected according to the formula provided in this manuscript. The same method is carried out for horizontal direction by taking width as a verge. Connected component testing is a very helpful method for working with binary pictures.

Ying (2011) stated that vertical and horizontal adjustment and picture improvement are carried out as pre-processing phases for character partitioning. Connected component testing is deployed in vertical and horizontal adjustment. Once, it is carried out these stages plate is converted to black characters or white backdrop and then resized to $100 \mathrm{X}$ 200. Thereafter, all characters are partitioned to a distinct size of $32 \times 32$.

Ter Brugge et al (1999) made use of picture binary and connected element marking techniques in their investigation. Ch. Jaya et al (2011) stated 3 matrices used in storing shield location and binary, number of columns and rows in black and white, respectively. The exact position of bottom and top limits are recognized, followed by upright projection and verging to partition the characters. Erdinc and Kursat (2011) deployed median filtering and contrast extension and blob coloring techniques for character partitioning. Contrast extension is useful in making picture shapes. Although the authors projected histogram equalization as a popular method to improve the appearance of the poor contrasted picture. Whereas median filtering removed unwanted noisy areas from the image. And blob coloring technique applies to binary images to recognize the contactless and closed area.

The blob coloring technique uses an L-shaped as the template to scan a picture from right to left and from top to bottom. A scanning procedure is deployed to detect the independent areas by acquiring the links into 4 dimensions from zero backdrops. The 4 dimensions blob coloring algorithm is used to the binary coding authorize shield picture for isolating the characters. The numbers are partitioned in the size of $28 \times 35$ at the end of the process and characters are partitioned in the size of $30 \times 40$. Lakshmi Narayana et al. (2012) proposed an extra algorithm centered on blob recognition. The character partitioning procedure comprises character elevation predicted, character breadth prediction, and blob isolation.

Character elevation prediction consists of 3 parts namely; color overturns, upright edge recognition, and horizontal estimation histogram. Color overturn helps in making the color of license shield characters black utilizing arithmetical analysis of edges. While 
upright edge recognition is deployed to recognize complete number plate. Sobel mark and picture binary algorithms are deployed to carry it out. A horizontal estimation histogram is deployed to identify the bottom and top limits of the character.

Table 1: List of countries that ANPR is supplied and reference

\begin{tabular}{|l|c|}
\hline A country that ANPR is Supplied & Reference \\
\hline European & Christo et al. (2006) \\
\hline USA, Singapore, China, Australia, South Africa & Jianbin et al. (2009) \\
\hline India & Prathamesh et al. (2009) \\
\hline China & Zhen-Xue et al. (2009) \\
\hline $\begin{array}{l}\text { Nigeria, Denmark, Cyprus, Germany, Finland, } \\
\text { Estonia, France, India, Norway, Portugal, Slovakia, } \\
\text { U.S.A, Czech Republic, Bulgaria }\end{array}$ & Roy and Ghoshal, 2011 \\
\hline Dutch & Ter Brugge and Jhuis (1999) \\
\hline Bulgaria, Isreal & Kang (2009) \\
\hline Korea & Nicolas et al. (2011) \\
\hline Muti-country & Donepudi, (2014a) \\
\hline Turkey & Yang et al. (2011) \\
\hline Australia & Morteza and Seyed (2011) \\
\hline Iran & $\begin{array}{c}\text { Francisco et al. (2012), Morteza } \\
\text { and Seyed (2011) }\end{array}$ \\
\hline USA & Francisco et al. (2012) \\
\hline
\end{tabular}

\section{CONCLUSION}

So far, it is obvious that automatic number plate recognition is a challenging structure due to the diverse number of stages and it is impossible to implement a hundred percent precision as each stage depends on the other. Also, some variables like diverse illumination situations, car shadow, and non-homogeneous size of license shield characters, fonts, and backdrop color affect the optimization of automatic number plate recognition. In the same vein, some structures still work in these constraint situations with the possibility of not yielding accurate outcomes. Also, some systems are customized to a specific location and country and such cases are presented in Table 1 . This study has given inclusive investigation of up-to-date advances and prospect trends in automatic number plate recognition that might be of great help to researchers who are involved in such developments.

\section{REFERENCES}

Anton, S.P. and Ariff, I. 2008. A Study of Car Park Control System Using Optical Character Recognition. International Conference on Computer and Electrical Engineering. pp. 866-870.

Ballard, D.H. (1981). Generalizing the Hough Transform to Detect Arbitrary Shapes. Pattern Recognition, 13(2):111-122.

ChJaya, L., Rani, A.J., Sri, K. R. and KantiKiran, M. 2011. "A Novel Approach for Indian License Recognition System," International Journal of Advanced Engineering Sciences and Technologies, 6(1):10-14.

Christos, N.E. Anagnostopoulos, I.E., Anagnostopoulos, I.D., Psoroulas, V. and Eleftherios, K. (2008). License Plate Recognition From Still Images and Video Sequences: A Survey, 9(3): 377-391. 
Cynthia, L., Julie, H., Breanne, C., Christopher, S. K. and Linda, M. (2011) License plate reader (LRP) police patrols in crime hot spots: an experimental evaluation in two adjacent jurisdictions. Journal of Experimental Criminology, Springer Netherlands, pp. 321-345, 2011.

Donepudi, P. K. (2014a). Technology Growth in Shipping Industry: An Overview. American Journal of Trade and Policy, 1(3), 137-142. https://doi.org/10.18034/ajtp.v1i3.503

Donepudi, P. K. (2014b). Voice Search Technology: An Overview. Engineering International, 2(2), 91-102. https://doi.org/10.18034/ei.v2i2.502

Erdinc, K.H. and K. Kursat, C.K. 2011. Artificial neural networks based vehicle license plate recognition. Procedia Computer Science, 3, 1033-1037.

Feng, W. 2008. Fuzzy-based algorithm for color recognition of license plates. Pattern Recognition Letters, 29(7): 1007-1020.

Fikriye, Ö. and Figen, Ö. 2012. "A New License Plate Recognition System Based on Probabilistic Neural Networks," Procedia Technology, vol. 1, pp. 124-128.

Francisco, M., Oliveira-Neto, L., Han, D. and Myong, K. J. 2012. "Online license plate matching procedures using license-plate recognition machine and new weighted edit distance," Transportation Research Part C: Emerging Technologies, 21(1): 306-320.

Hui, W. and Bing, L. (2011). License Plate Recognition System, in International Conference on Multimedia Technology (ICMT), , pp. 5425-5427.

Hui, W. and Bing, L. 2011. "License Plate Recognition System," in International Conference on Multimedia Technology (ICMT), pp. 5425-5427.

Jian, L., Dementhon, D. and Doermann, D. 2008. "Geometric Rectification of Camera- Captured Document Images," IEEE Transactions on Pattern Analysis and Machine Intelligence, vol. 9, no. 3, pp. 591-605.

Jianbin, J., Qixiang, Y. and Qingming, H. 2009. "A configurable method for multi-style license plate recognition," Pattern Recognition, 42(3): 358-369.

Kang, D. J. A. 2009. "Dynamic programming-based method for extraction of license numbers of speeding vehicles on the highway," International Journal of Automotive Technology, pp. 205-210.

Kaushik, D., Andrey, V., Jung-Won, K. and Kang-Hyun, J. 2010. "Vehicle license plate tilt correction based on the straight line fitting method and minimizing the variance of coordinates of projection point," International Journal of Control, Automation and Systems. pp. 975-984.

Kaushik, D., Ibrahim, K., Anik, S. and Kang-Hyun, J. 2012. An Efficient Method of Vehicle License Plate Recognition Based on Sliding Concentric Windows and Artificial Neural Network," Procedia Technology, 4, 812-819.

Lakshmi Narayana S., Suneetha Devi J., Bhargav Reddy I., Harish Paruchuri. (2012). Optimizing Voice Recognition using Various Techniques. CiiT International Journal of Digital Signal Processing, 4(4), 135-141

Lucjan, J. 2012. Quality assessment for a visual and automatic license plate recognition," Multimedia Tools and Applications Springer US, pp. 1-18.

Mei-Sen, P., Jun-Biao, Y. and Zheng-Hong, X. 2008. "Vehicle license plate character segmentation," International Journal of Automation and Computing, pp. 425-432.

Morteza, Z. and Seyed, M. S. (2011). License plate recognition system based on SIFT features. Procedia Computer Science, 3, 998-1002. 
Movva, L., Kurra, C., Koteswara Rao, G., Battula, R. B., Sridhar, M., \& Harish, P. (2012). Underwater Acoustic Sensor Networks: A Survey on MAC and Routing Protocols. International Journal of Computer Technology and Applications, 3(3).

Neogy, T. K., \& Paruchuri, H. (2014). Machine Learning as a New Search Engine Interface: An Overview. Engineering International, 2(2), 103-112. https://doi.org/10.18034/ei.v2i2.539

Nicolas, T., Antoine, V., Lionel, R. and Serge, M. 2011. "A cognitive and video-based approach for multinational License Plate Recognition," Machine Vision and Applications, SpringerVerlag, pp. 389-407.

Prathamesh, K., Ashish, K., Prateek, B. and Kushal, S. 2009. "Automatic Number Plate Recognition (ANPR)," in RADIOELEKTRONIKA. 19th International Conference.

Prathamesh, K., Ashish, K., Prateek, B. and Kushal, S. 2009. "Automatic Number Plate Recognition (ANPR)," in RADIOELEKTRONIKA. 19th International Conference, 2009.

Roy, A. and Ghoshal, D.P. 2011. Number Plate Recognition for use in different countries using an improved segmentation," in 2nd National Conference on Emerging Trends and Applications in Computer Science(NCETACS), pp. 1-5.

Shen-Zheng, W. and Hsi-Jian, L. 2002. "A cascade framework for real-time statistical plate recognition system," IEEE Trans. Inf. Forensics Security, 2(2): 267-282.

Shen-Zheng, W. and Hsi-Jian, L. 2007. "A cascade framework for real-time statistical plate recognition system," IEEE Trans. Inf. Forensics Security, 2(2): 267-282, 2007.

Suresh, K.V. Mahesh, G. K. and Rajagopalan, A.N. 2007. "Superresolution of license plates in real traffic videos," IEEE Trans. Intell. Transp. Syst, 8(2): 321-331.

Suresh, K.V., Mahesh Kumar, G. and Rajagopalan, A.N. 2007. Superresolution of license plates in real traffic videos. IEEE Trans. Intell. Transp. Syst, 8(2): 321-331.

Ter Brugge, M.H., Jhuis, J.A., Spaanenburg, L. and Stevens, J.H. 1999. "CNN- Applications in Toll Driving," Journal of VLSI signal processing systems for signal, image and video tehnology, pp. 465-477.

Ujwala, D., Ram Kiran, D. S., Jyothi, B., Fathima, S. S., Paruchuri, H., Koushik, Y. M. S. R. (2012). A Parametric Study on Impedance Matching of A CPW Fed T-shaped UWB Antenna. International Journal of Soft Computing and Engineering, 2(2), 433-436.

Vadlamudi, S. (2015). Enabling Trustworthiness in Artificial Intelligence - A Detailed Discussion. Engineering International, 3(2), 105-114. https:// doi.org/10.18034/ei.v3i2.519

Wenjing, J., Huaifeng, Z. and Xiangjian, H. 2007. "Region-based license plate detection," Journal of Network and Computer Applications, 30(4): 1324-1333.

Xin, F. and Guoliang, F. 2009. "Graphical Models for Joint Segmentation and Recognition of License Plate Characters," IEEE Signal Processing Letters, 16(1): 10-13.

Xing, Y., Xiao-Li, H. and Gang, Z. 2012. "License plate location based on trichromatic imaging and color discrete characteristic," Optik- International Journal for Light and Electron Optics, 123(16): 1486-1491.

Xing, Y., Xiao-Li, H. and Gang, Z. 2012. "License plate location based on trichromatic imaging and color discrete characteristic," Optik- International Journal for Light and Electron Optics, vol. 123(160: 1486- 1491.

Yang, Y. Xuhui, G. and Guowei, Y. 2011. "Study the Method of Vehicle License Locating Based on Color Segmentation," Procedia Engineering, 15, 1324-1329. 
Yifan, Z., Han, H., Zhenyu, Xu., Yiyu, H. and Shiqiu, L. 2011. "Chinese-style Plate Recognition Based on Artificial Neural Network and Statistics," Procedia Engineering, vol. 15, pp. 35563561.

Ying, W. 2011."An Algorithm for License Plate recognition Applied to Intelligent Transportation System," IEEE Transactions of Intelligent Transportation Systems, pp. 1-16.

You-Shyang, C. and Ching-Hsue, C. 2010. A Delphi-based rough sets fusion model for extracting payment rules of vehicle license tax in the government sector. Expert Systems with Applications, 37(3): 2161-2174.

Zhen-Xue, C., Cheng-Yun, L., Fa-Liang, C. and Guo-You, W. 2009. "Automatic License-Plate Location and Recognition Based on Feature Salience," IEEE Transactions on Vehicular Technology, 58(7): 3781-3785. 